\title{
Dual-Modality Activity-Based Probes as Molecular Imaging Agents for Vascular Inflammation
}

\author{
Nimali P. Withana*1, Toshinobu Saito*2, Xiaowei Ma*3, Megan Garland ${ }^{1}$, Changhao Liu ${ }^{3}$, Hisanori Kosuge ${ }^{2}$, \\ Myriam Amsallem ${ }^{2}$, Martijn Verdoes ${ }^{1}$, Leslie O. Ofori ${ }^{1}$, Michael Fischbein ${ }^{4}$, Mamoru Arakawa ${ }^{4}$, Zhen Cheng ${ }^{3}$, \\ Michael V. McConnell ${ }^{2}$, and Matthew Bogyo ${ }^{1,5}$ \\ ${ }^{I}$ Department of Pathology, Stanford University School of Medicine, Stanford, California; ${ }^{2}$ Department of Medicine (Cardiovascular), \\ Stanford University School of Medicine, Stanford, California; ${ }^{3}$ Department of Radiology, Stanford University School of Medicine, \\ Stanford, California; ${ }^{4}$ Department of Cardiothoracic Surgery, Stanford University School of Medicine, Stanford, California; and \\ ${ }^{5}$ Department of Microbiology and Immunology, Stanford University School of Medicine, Stanford, California
}

Macrophages are cellular mediators of vascular inflammation and are involved in the formation of atherosclerotic plaques. These immune cells secrete proteases such as matrix metalloproteinases and cathepsins that contribute to disease formation and progression. Here, we demonstrate that activity-based probes (ABPs) targeting cysteine cathepsins can be used in murine models of atherosclerosis to noninvasively image activated macrophage populations using both optical and PET/CT methods. The probes can also be used to topically label human carotid plaques demonstrating similar specific labeling of activated macrophage populations. Methods: Macrophage-rich carotid lesions were induced in FVB mice fed on a high-fat diet by streptozotocin injection followed by ligation of the left common carotid artery. Mice with carotid atherosclerotic plaques were injected with the optical or dual-modality probes BMV109 and BMV101, respectively, via the tail vein and noninvasively imaged by optical and small-animal $\mathrm{PET} / \mathrm{CT}$ at different time points. After noninvasive imaging, the murine carotid arteries were imaged in situ and ex vivo, followed by immunofluorescence staining to confirm target labeling. Additionally, human carotid plaques were topically labeled with the probe and analyzed by both sodium dodecyl sulfate polyacrylamide gel electrophoresis and immunofluorescence staining to confirm the primary targets of the probe. Results: Quantitative analysis of the signal intensity from both optical and PET/CT imaging showed significantly higher levels of accumulation of BMV109 and BMV101 $(P<0.005$ and $P<0.05$, respectively) in the ligated left carotid arteries than the right carotid or healthy arteries. Immunofluorescence staining for macrophages in cross-sectional slices of the murine artery demonstrated substantial infiltration of macrophages in the neointima and adventitia of the ligated left carotid arteries compared with the right. Analysis of the human plaque tissues by sodium dodecyl sulfate polyacrylamide gel electrophoresis confirmed that the primary targets of the probe were cathepsins X, B, S, and L. Immunofluorescence labeling of the human tissue with the probe demonstrated colocalization of the probe with CD68, elastin, and cathepsin S, similar to that observed in the experimental carotid inflammation murine model. Conclusion: We demonstrate that ABPs targeting the cysteine cathepsins can be used in

Received Dec. 18, 2015; revision accepted Apr. 14, 2016.

For correspondence or reprints contact either of the following:

Matthew Bogyo, Stanford University School of Medicine, Department of

Pathology, 300 Pasteur Dr., Stanford, CA 94305-5324.

E-mail: mbogyo@stanford.edu

Zhen Cheng, Stanford University School of Medicine,Department of Radiology, 1201 Welch Rd. Lucas Center, P095, Stanford, CA 94305-5484.

E-mail: zcheng@stanford.edu

${ }^{*}$ Contributed equally to this work.

Published online May 19, 2016.

COPYRIGHT (C) 2016 by the Society of Nuclear Medicine and Molecular Imaging, Inc. murine models of atherosclerosis to noninvasively image activated macrophage populations using both optical and PET/CT methods. The probes could also be used to topically label human carotid plaques demonstrating similar specific labeling of activated macrophage populations. Therefore, ABPs targeting the cysteine cathepsins are potentially valuable new reagents for rapid and noninvasive imaging of atherosclerotic disease progression and plaque vulnerability.

Key Words: animal imaging; optical; PET; PET/CT; vascular; activity-based probes; atherosclerosis; cathepsins; inflammation; macrophages

J Nucl Med 2016; 57:1583-1590

DOI: 10.2967/jnumed.115.171553

$A$ therosclerosis is a chronic cardiovascular disease characterized by plaque build-up within the arterial wall $(1,2)$. These plaques can remain asymptomatic for prolonged periods of time, yet subsequently rupture, leading to thrombus formation and myocardial infarction or stroke $(3,4)$. Therefore, the overall vulnerability (likelihood of rupture) of a plaque is a key parameter to predict disease progression and clinical sequelae and to help guide intervention and treatment strategies. A major clinical challenge is to identify specific molecular drivers of atherosclerosis disease pathology that could be used for diagnostic and treatment purposes.

Extensive studies over the past decade have provided substantial evidence that the extent of inflammation and angiogenesis within a plaque are directly linked to its risk of rupture $(5,6)$. Among inflammatory cells within the plaque, macrophages appear to play a major role. In early atherosclerotic stages, blood-derived monocytes are recruited from the lumen to the subendothelial space of the arterial wall, where they accumulate and differentiate into macrophages $(7,8)$. Monocyte-derived macrophages are key cellular mediators of atherosclerotic inflammation. They produce proteases such as matrix metalloproteinases and cysteine cathepsins that participate in extracellular matrix remodeling and destabilization of atherosclerotic plaques $(9,10)$. Therefore, molecular probes of activated macrophages offer the possibility to measure the overall vulnerability of plaques.

Most cardiovascular imaging modalities used in clinical practice, such as coronary angiography, CT angiography, and MRI, mainly provide anatomic data on the presence and size of plaques. Additionally, approved clinical contrast agents, such as indocyanine 
green, have been traditionally used to assess blood flow and the structural integrity of the endothelial lining of vasculature. Recent reports have taken advantage of the lipophilicity of the indocyanine green molecule to show uptake of dye by lipid-rich atherosclerotic plaques (11). However, the drawback of these optical-only contrast agents is that tissue penetrance limits dye detection to $5-10 \mathrm{~mm}$, severely limiting the ability of these dyes to be used for noninvasive imaging, and they do not provide any information about the inflammatory state of a given plaque (12). To begin to address the problem of noninvasively detecting vulnerable plaques likely to rupture, several new imaging modalities aimed at targeting molecular and cellular activities have emerged $(13,14)$. PET/CT has been explored as a technique to detect and quantify the presence of inflammation within carotid plaques (13-18). Most PET/CT studies for atherosclerosis make use of ${ }^{18} \mathrm{~F}-\mathrm{FDG}$ as a contrast agent. This agent accumulates in cells with high metabolic activity, such as inflammatory cells, and provides a readout of inflammatory infiltrates in atherosclerotic plaques $(17,19,20)$. However, its overall high myocardial background levels and nonspecific mechanism of action have limited the implementation of this contrast agent in cardiovascular disease. For example, ${ }^{18} \mathrm{~F}$-FDG cannot be used in patients with uncontrolled diabetes mellitus, because the uptake of ${ }^{18} \mathrm{~F}-\mathrm{FDG}$ is competed by the significantly elevated levels of plasma glucose. Additionally, increased background signal in the heart due to the elevated metabolic activity of the myocardium is a significant drawback for imaging coronary plaques $(21)$.

Efforts toward developing more specifically targeted agents include the use of Arg-Gly-Asp (RGD) peptide tracers (21,22). This short RGD peptide binds to the cell surface glycoprotein receptor $\alpha_{v} \beta_{3}$ integrin, found on endothelial cells and macrophages. RGD peptides have been developed into PET/CT radiotracers by conjugation to ${ }^{18} \mathrm{~F}-$ galacto or ${ }^{68} \mathrm{Ga}$-DOTA (23). However, this approach is limited in that it reports on levels of all cells expressing this target receptor and is not specific to activated macrophages $(24,25)$.

Macrophages within a developing plaque secrete proteases that function as regulators of the overall atherosclerotic disease pathology. Therefore, agents that can provide a direct readout of protease activity hold promise as contrast agents for vascular inflammation imaging (13). One class of proteases that are highly upregulated in activated macrophages is the cysteine cathepsins. These proteases have been shown to play important roles in extracellular matrix remodeling and are implicated in the development and progression of atherosclerosis (26). For example, cathepsin S has been shown to colocalize with a major protein of the extracellular matrix, elastin, in the arterial medium of atheromas (27), especially in regions of elastin breaks (28). Several cysteine cathepsin-specific probes have been developed for both optical and PET imaging of tumor progression and lung inflammation (29-31). However, cathepsin protease probes equipped with both optical and PET tracers have never been tested in models of atherosclerosis.

In this study, we investigate the use of both a quenched fluorescent cathepsin ABP (BMV109) and a dual-modality optical, PET/CT probe (BMV101) to image activated macrophages in an experimental murine model of carotid inflammation. Cellular studies using in situ and ex vivo optical methods were also performed and confirm that probes are highly specific and that they can provide an accurate readout of levels of these proteases that correlates with disease severity. In concurrence with previous studies $(27,28)$, probe labeling also colocalized with elastin in carotid samples. We also demonstrate the use of the probes for detecting macrophage-derived cathepsins in human carotid endarterectomy specimen by topical application of the probe to the tissue samples. These results obtained in the experimental mouse model are consistent with what occurs in the human disease with respect to levels of cathepsins in activated macrophages. Our study provides evidence that cysteine cathepsin ABPs have the potential to be used for noninvasive imaging of atherosclerotic plaque inflammation to allow better patient stratification and identification of vulnerable inflammatory plaques at high risk of rupture and atherothrombotic events.

\section{MATERIALS AND METHODS}

\section{Animals and Model Induction}

Macrophage-rich carotid lesions were induced in FVB mice as described previously $(22,32)$. Briefly, 8 -wk-old mice were fed a highfat diet (HFD) for $4 \mathrm{wk}$. After $1 \mathrm{mo}$ on the diet, diabetes was induced by 5 daily intraperitoneal injections of streptozotocin (STZ; $40 \mathrm{mg} / \mathrm{kg}$, Sigma-Aldrich). Two weeks after the initiation of STZ injection, the left common carotid artery was ligated below the bifurcation with 5-0 silk ligature (Ethicon) under $2 \%$ inhaled isoflurane (ligation group), to develop macrophage-rich neointimal proliferation. The wound was closed by suture, and the animals recovered on a warming blanket. Eight animals were used for each time point, divided equally between control and diseased groups. All animal procedures were approved by the Administrative Panel on Laboratory Animal Care at Stanford University, CA.

\section{In Vivo Fluorescence Imaging}

Two weeks after the surgery, the mice were imaged noninvasively by fluorescence molecular tomography (FMT) (FMT 2500 imaging system; Visen) at 680/700 nm excitation/emission wavelength. Imaging was performed before and then $4 \mathrm{~h}$ after injection of the probe BMV109 at a dose of $10 \mathrm{nM}$ in $10 \%$ dimethyl sulfoxide/phosphate-buffered saline (PBS) via the tail vein. After in vivo fluorescence imaging, the left and right carotid arteries were surgically exposed and in situ fluorescence imaging was performed on the Maestro imaging system (Cri) at 675/690 nm excitation/emission. The carotid arteries and aortic arch were then removed en bloc and imaged ex vivo using Maestro. Images were analyzed by placing regions of interest over the carotid arteries and calculating average signal intensity divided by exposure time.

\section{Radiolabeling}

Briefly, ${ }^{64} \mathrm{Cu}-\mathrm{BMV} 101$ was prepared by incubation of $2 \mu \mathrm{L}$ of BMV101 $(10 \mathrm{mM})$ in $90 \mu \mathrm{L}$ of sodium acetate buffer $(0.1 \mathrm{M}, \mathrm{pH}$ 5.5) with $37 \mathrm{MBq}(4 \mathrm{mCi})$ of ${ }^{64} \mathrm{CuCl}_{2}$ in $100 \mu \mathrm{L}$ of sodium acetate buffer $(0.1 \mathrm{M}, \mathrm{pH} 5.5)$ at $37^{\circ} \mathrm{C}$ for $1 \mathrm{~h}$. After being cooled to room temperature, the reaction mixture was then purified by reversed-phase high-performance liquid chromatography (HPLC), with the mobile phase starting from $95 \%$ solvent $\mathrm{A}$ (di-water with $0.1 \%$ trifluoroacetic acid) and 5\% solvent B (acetonitrile with $0.1 \%$ trifluoroacetic acid) for $3 \mathrm{~min}$ to $5 \%$ solvent $\mathrm{A}$ and $95 \%$ solvent $\mathrm{B}$ at $23 \mathrm{~min}$. The eluted fractions containing ${ }^{64} \mathrm{Cu}-\mathrm{BMV} 101$ (retention time, $18.9 \mathrm{~min}$ ) were then collected. The collection was diluted 10 times with di-water and then passed through a $\mathrm{C} 18$ light cartridge (Waters). After being generally washed with $10 \mathrm{~mL}$ of di-water, the ${ }^{64} \mathrm{Cu}-\mathrm{BMV} 101$ was eluted from the cartridge with $300 \mu \mathrm{L}$ of $80 \%$ ethanol. The product was then reconstituted in $3 \mathrm{~mL}$ of $0.9 \%$ saline and passed through a $0.22-\mu \mathrm{m}$ Millipore filter into a sterile vial for animal PET/CT imaging.

\section{PET/CT In Vivo Imaging}

Mice were intravenously injected with $3.7 \mathrm{MBq}(100 \mu \mathrm{Ci})$ of ${ }^{64} \mathrm{Cu}-$ BMV101 and imaged after 4 and $24 \mathrm{~h}$ using an Inveon small-animal PET/CT scanner (Siemens). Briefly, a CT anatomic image scan was acquired $(80 \mathrm{kV}, 500 \mu \mathrm{A})$ with a pixel size of approximately $0.1 \mathrm{~mm}$. After CT imaging, whole-body PET imaging was performed with 5-min static scanning. The PET images were reconstructed using the 
ordered-subsets expectation maximization 3-dimensional algorithm based on CT attenuation and analyzed using the Inveon Research Workplace software (Siemens). PET voxel size was $0.80 \times 0.86 \times$ $0.86 \mathrm{~mm}$, for a total of $128 \times 128 \times 159$ voxels. PET/CT images were analyzed, and tissue radioactivity was calculated and expressed as decay-corrected percentage injected dose per gram of tissue (\%ID/g). After the 4- and 24-h PET/CT imaging, the left and right carotid arteries were surgically exposed and then removed for in situ and ex vivo fluorescence imaging, as described above. Investigators conducting the study were masked to which groups were being imaged.

\section{Biodistribution}

After PET/CT imaging, the mice were anesthetized and sacrificed. All organs were collected, weighed, and measured with a $\gamma$-counter. The uptake of each organ was calculated and calibrated with decaycorrection and expressed as \% ID/g.

\section{Ex Vivo Human Carotid Artery Plaque Analysis}

Human carotid plaques were collected from endarterectomy procedures by the Division of Vascular Surgery of Stanford, after approval of Stanford University IRB (protocol 22141). Fresh carotid artery plaques were removed en bloc to preserve plaque structure. After collection, specimens were immediately put in PBS, cooled on ice, and processed. Each plaque was randomly divided in half. One section was used for sodium dodecyl sulfate polyacrylamide gel electrophoresis (SDS-PAGE) analysis as described below to confirm the primary targets of the cathepsin probe. The second section was embedded in optimal-cutting-temperature compound and microcut into 5- $\mu \mathrm{m}$ slices, before topical application of $1 \mu \mathrm{M}$ of the optical probe BMV109, along with immunofluorescence staining of elastin, cathepsin $S$, or the macrophage marker CD68.

\section{SDS-PAGE Analysis of Human Carotid Artery Plaque}

The human carotid artery tissues were sonicated in muscle lysis buffer (1\% Triton X-100 [Sigma]; 0.1\% SDS, 0.5\% sodium deoxycholate, $4 \mathrm{mM}$ dithiothreitol, PBS [pH 5.5]), and protein concentration was determined using a BCA kit (Pierce ThermoFisher Scientific). Aliquots of $50 \mu \mathrm{g}$ of total protein were treated either with or without the cathepsin inhibitor GB111- $\mathrm{NH}_{2}(33)$ at a concentration of $100 \mu \mathrm{M}$ and samples incubated for $1 \mathrm{~h}$ at $37^{\circ} \mathrm{C}$. Samples were then incubated with the probe BMV109 from a $100 \times$ dimethyl sulfoxide stock solution, yielding a final dimethyl sulfoxide concentration of $1 \%$. Samples were incubated for $30 \mathrm{~min}$ at $37^{\circ} \mathrm{C}$ and then solubilized with $4 \times$ sample buffer. Proteins were resolved by $12 \%$ SDS-PAGE and scanned using a Typhoon flatbed laser scanner (excitation $633 \mathrm{~nm} / \mathrm{emission} 670 \mathrm{~nm}$ )

\section{Immunofluorescence Staining}

Briefly mouse carotid arteries were cut into 2 three-millimeter sections. These sections were embedded immediately in optimalcutting-temperature compound (Sakura Finetek USA, Inc.) and flashfrozen in liquid nitrogen. Frozen sections $(5-\mu \mathrm{m}$ thick) were fixed in acetone for $10 \mathrm{~min}$ at $-20^{\circ} \mathrm{C}$. After being washed in PBS, the sections were incubated with macrophage marker antimouse CD68 (1:1,000; catalog no. MCA1957 [AbD Serotec]), antielastin (1:50; catalog no. bs-11057R [One World Lab]), or anticathepsin S (1:200; catalog no. bs-8558R [One World Lab]) antibodies overnight at $4^{\circ} \mathrm{C}$. The primary antibodies were detected with Alexa Fluor 488-conjugated antirat IgG and Alexa Fluor 594-conjugated antirabbit IgG (Molecular Probes) at room temperature for $1 \mathrm{~h}$. Finally, sections were stained with $4^{\prime}, 6-$ diamidino-2-phenylindole (DAPI) and fluorescence images acquired by confocal microscopy. Sections were imaged by tile scan and at $20 \times$ using a Zeiss Axiovert $200 \mathrm{M}$ confocal microscope in Cy5, fluorescein isothiocyanate (FITC), and Texas-Red channels.

\section{Topical Application of Activity-Based Probes}

Human carotid endarterectomy samples $(n=2)$ were collected from Stanford's vascular operating room and topically labeled with probe or an antibody of interest as described previously (34). Briefly, the fresh carotid tissue was frozen in optimal-cutting-temperature compound before sectioning. Sections $(5-\mu \mathrm{m}$ thick) were fixed for $10 \mathrm{~min}$ in acetone at $-20^{\circ} \mathrm{C}$, and sections were blocked in $1 \%$ blocking reagent (catalog no. FP1020; Perkin Elmer) for $1 \mathrm{~h}$ and then stained for $1 \mathrm{~h}$ with $1 \mu \mathrm{M}$ BMV109 in PBS. As a control for the probe labeling, serial tissue sections were first incubated with $100 \mu \mathrm{M}$ cathepsin inhibitor GB111- $\mathrm{NH}_{2}$ to block cysteine protease activity for $1 \mathrm{~h}$ at room temperature. Sections were washed in PBS $(3 \times 5 \mathrm{~min})$ and then incubated with probe $(1 \mu \mathrm{M}$ in PBS $)$ for $1 \mathrm{~h}$ at room temperature. Sections were washed in PBS $(3 \times 5 \mathrm{~min})$ and then stained with the following antihuman antibodies, macrophage marker CD68 (1:1,000; MCA1815T [BioRad]), elastin (1:50; catalog no. bs11057R [One World Lab]), or cathepsin S (1:200; catalog no. bs-8558R [One World Lab]) overnight at $4^{\circ} \mathrm{C}$. Slides were washed, and the primary antibodies were detected with Alexa Fluor 488-conjugated antirat IgG and Alexa Fluor 594-conjugated antirabbit IgG (Molecular Probes) at room temperature for $1 \mathrm{~h}$. Finally, sections were stained with DAPI and fluorescence images acquired by confocal microscopy. All sections were imaged by tile scan and at $20 \times$ using a Zeiss Axiovert $200 \mathrm{M}$ confocal microscope in Cy5, FITC, and Texas-Red channels. All images were taken using a multitrack channel acquisition to prevent emission crosstalk between fluorescent dyes. Single XY, XZ plane images were acquired in $1,024 \times 1,024$ resolution. Images were processed as separate channels using Huygens deconvolution software or ImageJ and merged as a single image. Mosaic images of fluorescence labeling were taken using $20 \times$ objective and stitched using $15 \%$ overlay.

\section{Statistical Analysis}

Statistics were performed using the data analysis package within GraphPad Prism 6.0 for Windows (GraphPad Software). Unless otherwise stated, tests comparing 2 means are Student $t$ tests, with equal variance assumed. Error bars indicate SEM unless otherwise stated. All data are expressed as mean \pm SEM. Comparisons between ligated (left) and nonligated (right) carotids for in situ and ex vivo fluorescence signal intensities were analyzed by the Wilcoxon signed-rank test. Comparisons of biodistribution of liver, spleen, kidney, and lung were analyzed by the unpaired Student $t$ test, as they were normally distributed. A $P$ value of less than 0.05 was considered statistically significant.

\section{RESULTS}

\section{Fluorescence Imaging of Murine Carotid Arteries Using Fluorescent Cathepsin Probes}

We have recently demonstrated that the cathepsin ABP containing a phenoxymethyl ketone electrophile, BMV109 (Fig. 1A), has enhanced in vivo properties and broad reactivity toward cathepsins X, B, S, and L $(35,36)$. Given the success of this optical probe in detecting cysteine cathepsins, and the reported role that these proteases have in atherosclerosis, we investigated the use of this probe to detect plaques in an experimental atherosclerotic murine model. We assessed the extent of probe accumulation by noninvasive fluorescence-mediated tomography (FMT) imaging in the carotid artery (Fig. 1).

FMT images showed probe signal enhancement in the left carotid artery $4 \mathrm{~h}$ after injection of BMV109 in mice with a ligated artery, with no signal observed in control mice (Fig. 1B). This was further confirmed by in situ imaging at $4 \mathrm{~h}$, where enhanced florescence signal was localized to the ligated left carotid artery compared with the nonligated right artery and control mouse (Fig. 1C). Ex vivo 


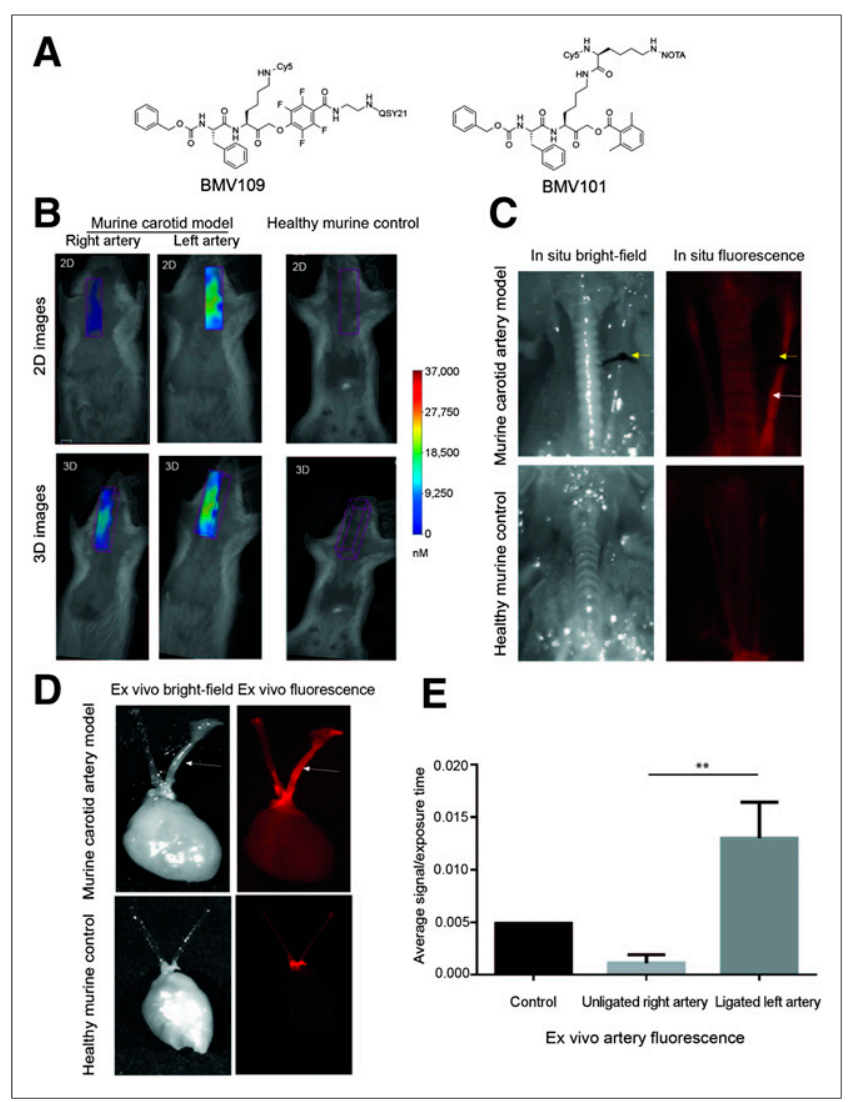

FIGURE 1. Application of activity-based probe BMV109 in experimental carotid inflammation model. (A) Structure of fluorescent cathepsin probe BMV109. (B) Noninvasive FMT imaging in murine carotid arteries and healthy control arteries. Both 2- and 3-dimensional images show high signal in left carotid artery of diseased mouse compared with right artery and control. (C) Corresponding in situ fluorescence imaging of BMV109 in murine carotid arteries and control healthy mouse. (D) Ex vivo florescence imagining of diseased and healthy carotid arteries. (E) Quantitative analysis of ex vivo fluorescence showed significantly higher signal in left ligated carotid artery than the nonligated carotid artery and control. $n=3,{ }^{\star \star} P<0.005$ by $t$ test.

imaging further demonstrated significantly higher signal from the left than right carotid arteries (Fig. 1D). Quantitative analysis of the signal intensity confirmed that the ligated left carotid arteries had significantly higher signal than the right carotid arteries $(0.01301 \pm 0.001974$ vs. $0.001108 \pm 0.0004618, P=0.0042$, Fig. 1E). Immunofluorescence staining for macrophages in crosssectional slices demonstrated substantial infiltration of macrophages in the neointima and adventitia of the ligated left carotid arteries (Fig. 2). In contrast, only a small number of macrophages were seen in the adventitia of the right (nonligated) carotid arteries, and no macrophage staining was observed in control arteries. Elastin remodeling was observed in both the left and the right arteries of the diseased model, with weak staining observed in the control healthy artery. Additionally, cathepsin S expression was confirmed in both arteries of the diseased mice but not in the control. The probe colocalized to areas of macrophage infiltration as well as elastin and cathepsin S expression in the neointima, highlighting regions of tissue remodeling and damage. Longitudinal cross-sections of the arteries also showed colocalization of the probe with macrophages in the neointima, along with colocalization with cathep$\sin \mathrm{S}$ and elastin, with higher signal observed in the left ligated carotid artery than the right (Supplemental Fig. 1; supplemental materials are available at http://jnm.snmjournals.org).

\section{Imaging of Carotid Arteries Using Dual-Modality Optical and PET/CT Cathepsin Probe}

We next applied the dual-modality optical/PET probe BMV101 (structure in Fig. 1A) that we recently showed to be an effective label of activated macrophages in mouse models and human clinical studies of lung fibrosis (Fig. 3A) (35). This reagent contains both the optical fluorophore and a chelator group that can be used for labeling with radionuclides. For these studies, we used ${ }^{64} \mathrm{Cu}$ because it has a relatively long half-life and allows imaging at late time points (i.e., $4 \mathrm{~h}$ and greater). The radiolabeling yield was higher than $90 \%$ (calculated from the HPLC). The radiochemical purity, defined as the ratio of the main product peak to the other peaks, was determined by radio-HPLC to be greater than $95 \%$, and the specific activity of the probe was determined to be 111-148 GBq (3-4 Ci)/mmol. We performed optical/PET/CT imaging studies at 4 and $24 \mathrm{~h}$. Similar to the results observed with the optical probe BMV109, we detected higher signal intensity from ${ }^{64} \mathrm{Cu}-$ BMV101 in the left ligated artery than the right (nonligated) and healthy control arteries (Fig. 3B). Quantitative analysis of the PET signal intensity showed that the ligated left carotid arteries had significantly higher signal than the right carotid arteries at both $4 \mathrm{~h}$ $(11.10 \pm 0.8842$ vs. $8.298 \pm 0.2605, P=0.0383)$ and $24 \mathrm{~h}(6.553 \pm$ 0.5843 vs. $4.589 \pm 0.2142, P=0.0343$, Fig. 3 C) after probe injection.

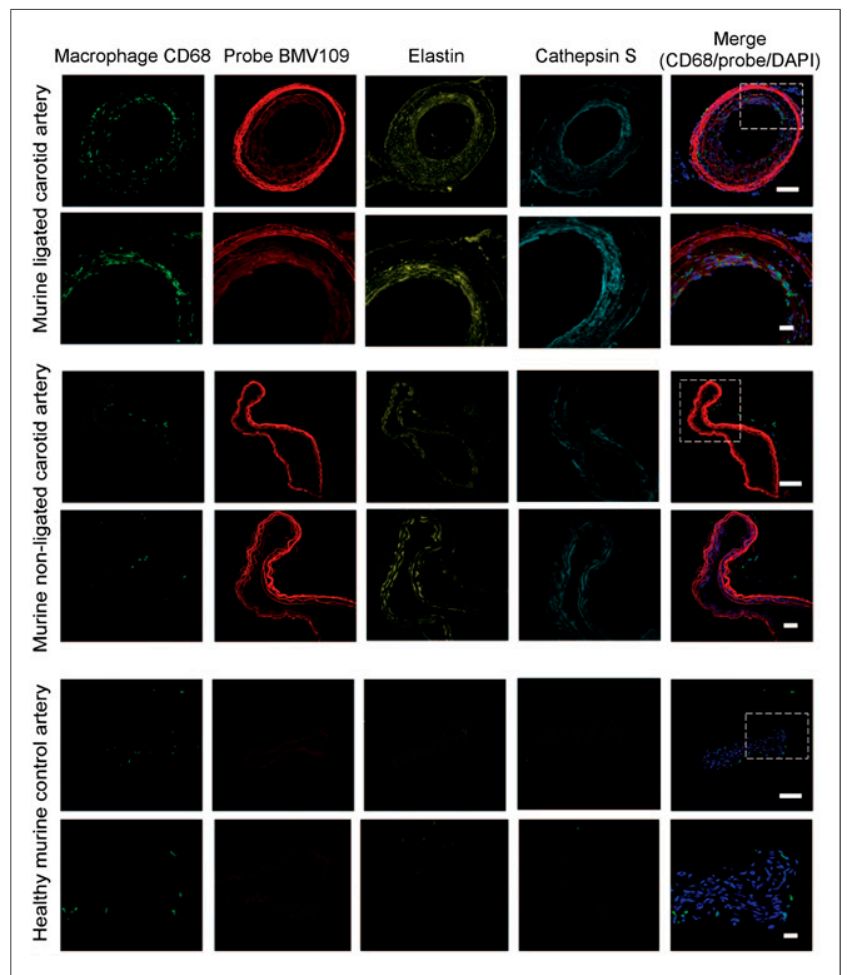

FIGURE 2. Immunostaining of representative carotid arteries. Tissue cross-sections from ligated, nonligated, and control carotid arteries were labeled with optical probe BMV109 (red) and costained with macrophage activation marker CD68 (green), elastin (yellow), and cathepsin $S$ (cyan). DAPI nuclear stain is shown in blue. Samples were tilescanned at high resolution to generate full images for which scale bar represents $1 \mathrm{~mm}$. White boxes on full images indicate region that higher magnification images were taken at $40 \times$. Scale bars on zoom images are $10 \mu \mathrm{m}$. 


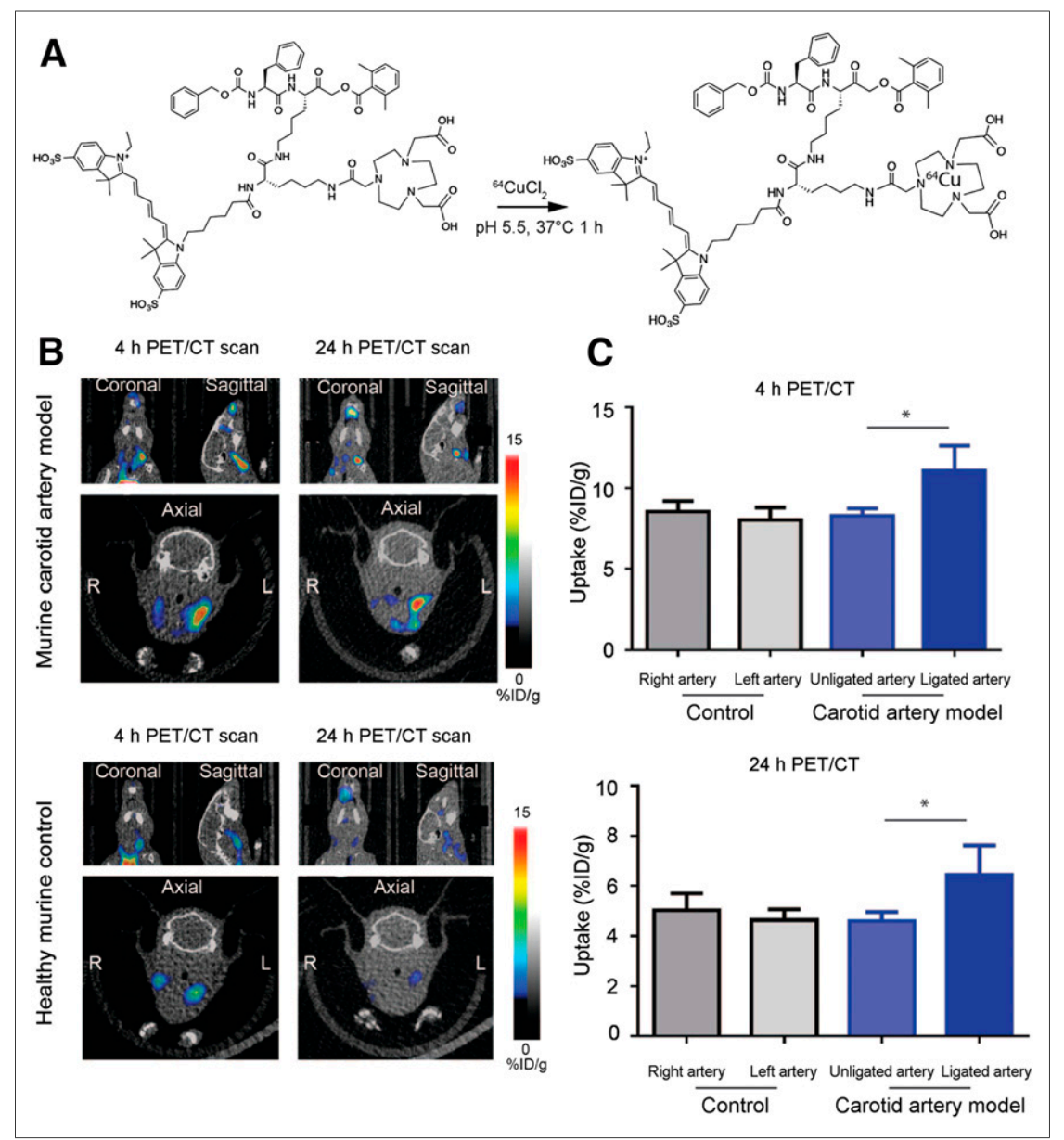

FIGURE 3. Application of dual optical/PET imaging probe ${ }^{64} \mathrm{Cu}-\mathrm{BMV} 101$. (A) Structure and labeling conditions for dual optical/PET probe BMV101. (B) Noninvasive PET/CT scans of mice with and without ligated carotid arteries. Coronal (top left), sagittal (top right), and axial (bottom, showing left and right). Images are shown for representative diseased and healthy mice imaged at 4 and $24 \mathrm{~h}$. (C) Quantification of 4- and 24-h PET/CT intensity from ligated, nonligated, and healthy carotid arteries of all mice. Error bars indicate mean + SEM, $n=3,{ }^{*} P<0.05$ by $t$ test.
As an additional control, we repeated the dual-modality optical/PET analysis with the addition of a cohort of mice that were fed a HFD but were not administered STZ. These mice also had their left common carotid artery ligated below the bifurcation and therefore should have all the same levels of blood pooling and general inflammation as the disease model but without the ability to generate plaques. We performed optical/PET/CT imaging studies at 4 and $24 \mathrm{~h}$ and detected higher signal intensity from ${ }^{64} \mathrm{Cu}$-BMV101 in the left ligated artery of HFD + STZ mice than in the left ligated artery of HFD-alone or the nonligated left artery of control mice (Fig. 5A). We also detected higher signal intensity from ${ }^{64} \mathrm{Cu}$-BMV101 in the left HFD + STZ ligated artery than in the right (nonligated), HFD (nonligated), and healthy control arteries (Fig. 5A). Quantitative analysis of the PET signal intensity showed that the ligated left carotid artery of HFD + STZ mice had significantly higher signal than the left control carotid artery at both $4 \mathrm{~h}(15.13 \pm 0.9034$ vs. $8.776 \pm 0.6816, P=0.0049)$ and $24 \mathrm{~h}$ $(7.653 \pm 0.6421$ vs. $4.448 \pm 0.3992, P=$ 0.0133 , Fig. 5B) after probe injection. Importantly, there was no significant difference observed between the ligated left carotid artery of HFD-only mice compared with controls at $4 \mathrm{~h} \mathrm{(12.11 \pm}$ 1.043 vs. $8.776 \pm 0.6816, P=0.0554)$ and $24 \mathrm{~h}(6.116 \pm 0.4761$ vs. $4.448 \pm$ $0.3992, P=0.0549)$. This confirms that the probe is activated by macrophagedriven inflammation in plaques that is not observed in arteries that had been ligated but that did not have plaques.

We examined the overall biodistribution of the probe in all organs at each time point (Supplemental Fig. 2A). The probe accumulated in the blood, liver, lung, and kidney but showed the most substantial increase in accumulation in the left carotid artery $(8.32 \% \mathrm{ID} / \mathrm{g}$ at $4 \mathrm{~h}$ or $6.39 \% \mathrm{ID} / \mathrm{g}$ at $24 \mathrm{~h})$ compared with the right $(6.49 \% \mathrm{ID} / \mathrm{g}$ at $4 \mathrm{~h}$ or $4.95 \% \mathrm{ID} / \mathrm{g}$ at $24 \mathrm{~h}$ ). The stability of ${ }^{64} \mathrm{Cu}-\mathrm{BMV} 101$ was further evaluated in PBS and mouse serum. As shown by the radio-HPLC analysis, ${ }^{64} \mathrm{Cu}-\mathrm{BMV} 101$ was highly stable, and there was no degradation observed in PBS buffer or after 4-h incubation in mouse serum at $37^{\circ} \mathrm{C}$ (Supplemental Fig. 2B). Because the probe has dual optical and PET labels, we were able to visualize the increase in optical probe signal in the left carotid artery compared with the right by in situ optical imaging and by ex vivo imaging of the arteries (Figs. 4A and B, respectively). Analysis of the ex vivo signal intensity showed that the ligated left carotid arteries had significantly higher BMV101 probe signal than the right carotid arteries $(0.02629 \pm 0.006184$ vs. $0.006500 \pm 0.0002774, P=$ 0.0330, Fig. 4C). Confocal microscopy further confirmed the elevated probe signal and colocalization of the probe with the macrophage marker CD68 in the diseased left carotid artery compared with the right carotid artery (Fig. 4D).
We also examined the overall biodistribution of the probe in all organs at $24 \mathrm{~h}$ (Supplemental Fig. 3). The probe accumulated in the blood, liver, lung, and kidney but showed the most significant increased accumulation in the left carotid artery of HFD + STZ mice compared with the HFD-alone or control groups. Finally, we were able to visualize the increase in optical probe signal in the left carotid artery of the HFD + STZ model compared with the HFD-alone and control by in situ optical imaging and by ex vivo imaging of the arteries (Supplemental Fig. 4A). Analysis of the ex vivo signal intensity showed that the ligated left carotid arteries of the HFD + STZ model had significantly higher BMV101 probe signal than the control left carotid arteries $(0.03949 \pm 0.007866$ vs. $0.008808 \pm 0.000645, P+$ 0.0177, Supplemental Fig. 4B). No significant difference was seen when comparing HFD with control.

\section{Topical Application of Activity-Based Probe on Human Carotid Artery Specimens}

Having shown the effective delivery of an optical and bimodal optical/PET imaging probe to visualize plaque formation in an experimental carotid inflammation model, we further validated these imaging probes for diagnostic use with human tissues. We and 


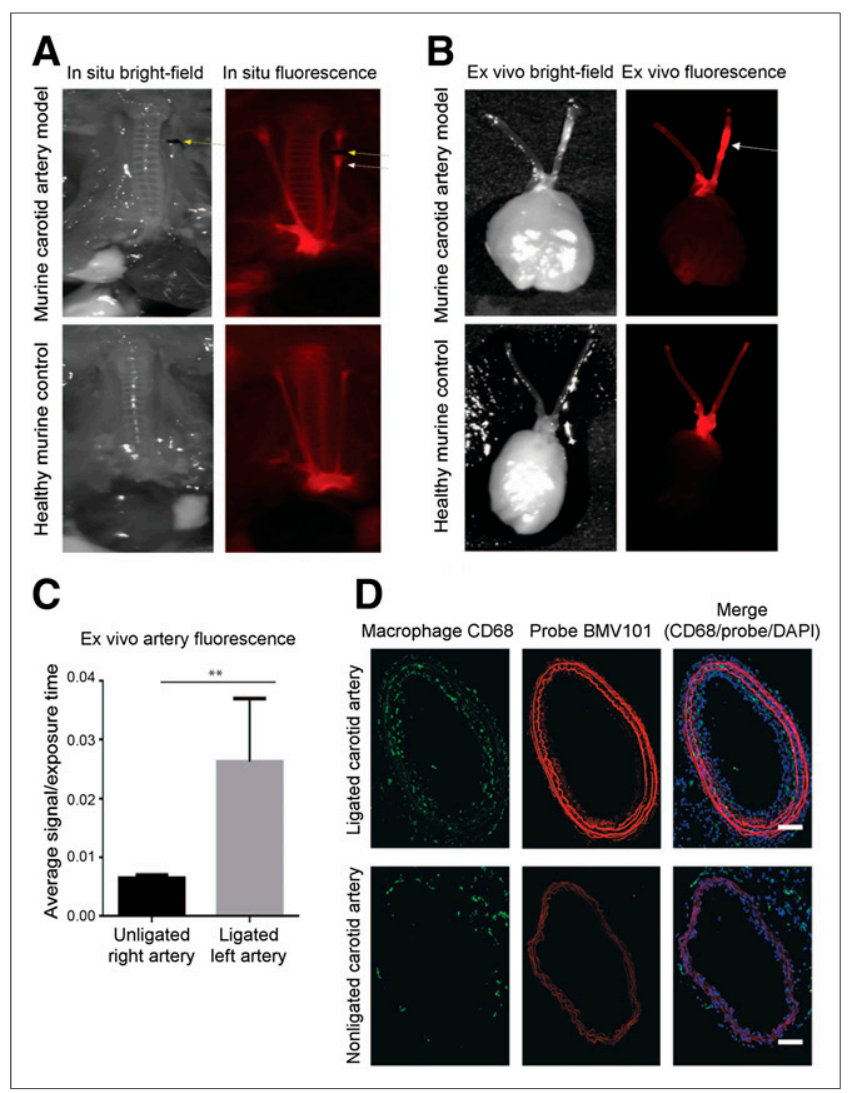

FIGURE 4. In situ, ex vivo, and immunostaining of representative carotid arteries from ${ }^{64} \mathrm{Cu}-\mathrm{BMV} 101-$ treated mice. (A) In situ fluorescence imaging of ${ }^{64} \mathrm{Cu}-\mathrm{BMV} 101$ in murine carotid arteries and control healthy mouse. (B) Ex vivo florescence imagining of diseased and healthy carotid arteries. (C) Quantitative analysis of ex vivo fluorescence showed significantly higher signal in left ligated carotid artery than nonligated carotid artery. $n=3$, ${ }^{*} P<0.05$ by $t$ test. (D) Tissue sections from ligated and nonligated carotid arteries were labeled with optical probe ${ }^{64} \mathrm{Cu}-\mathrm{BMV} 101$ (red) and costained with macrophage activation marker CD68 (green). DAPI nuclear stain is shown in blue. Samples were tile-scanned at high resolution to generate full images for which scale bar represents $1 \mathrm{~mm}$.

others have previously demonstrated that small-molecule ABPs can be used to topically label active cathepsins in excised tissues $(37,38)$. Two fresh human carotid endarterectomy specimens were split into sections to perform labeling using the optical probe BMV109 (Fig. 6A). Analysis of the plaque tissue by SDS-PAGE confirmed that the primary targets of the probe were cathepsins $\mathrm{X}$, $\mathrm{B}, \mathrm{S}$, and $\mathrm{L}$ and that labeling of these targets was blocked when the tissue lysate was preincubated with a cathepsin inhibitor GB111- $\mathrm{NH}_{2}$ (Fig. 6B). The second specimen of the carotid artery was embedded in optimal-cutting-temperature compound and sectioned before the probe was topically applied to the tissue sections, along with antibodies for elastin, cathepsin $\mathrm{S}$, and the macrophage marker CD68 (Fig. 6C). Similar to the results for the experimental carotid inflammation murine model, we observed colocalization of the probe with CD68, elastin, and cathepsin S. The probe signal was blocked when the tissue sample was pretreated with the cathepsin inhibitor GB111- $\mathrm{NH}_{2}$ (33) (Fig. 6C). These data confirmed that we could label fresh carotid plaques by topical application of the probe. Furthermore, probe signal, indicating active cathepsins, was found at sites of plaques, suggesting that cathepsins are likely to be useful biomarkers for monitoring disease activity.

\section{DISCUSSION}

Cardiovascular disease continues to be the leading cause of death worldwide. This statistic, coupled with the silent, and often asymptomatic, nature of atherosclerosis highlights the critical need for improved diagnostics that detect early-stage, asymptomatic at-risk cardiovascular lesions. Here, we show that the quenched fluorescent ABP BMV109 and the dual optical and PET/CT ABP BMV101 are efficacious as noninvasive imaging agents for atherosclerosis. In a murine model, we demonstrated the ability of BMV109 to detect atherosclerotic plaques using noninvasive FMT imaging. BMV109 also highlighted plaques when topically applied to fresh frozen tissue sections and imaged via confocal microscopy. We showed that the dual-labeled probe BMV101 could be used to noninvasively image plaques by both PET and optical detection methods. Further, the probe was able to distinguish plaques in the HFD + STZ model, a noninflammatory smooth muscle cell-rich restenosis lesion model from the HFD-alone model. Finally, we demonstrated the ability of BMV109 to detect plaques in a human carotid plaque sample ex vivo. Taken together, these data highlight the value of activity-based probes as potential noninvasive diagnostic tools to detect vascular inflammation.

This work adds to the growing body of research focused on targeted, noninvasive imaging agents for the detection of vulnerable atherosclerotic lesions. Such agents should improve our ability to provide more precise identification and prediction of clinical events. In this study, we used ABPs with optical reporters, as well as the dual optical and PET/CT reporters, to highlight atherosclerotic plaques. Because our probes target active cathepsins, signals

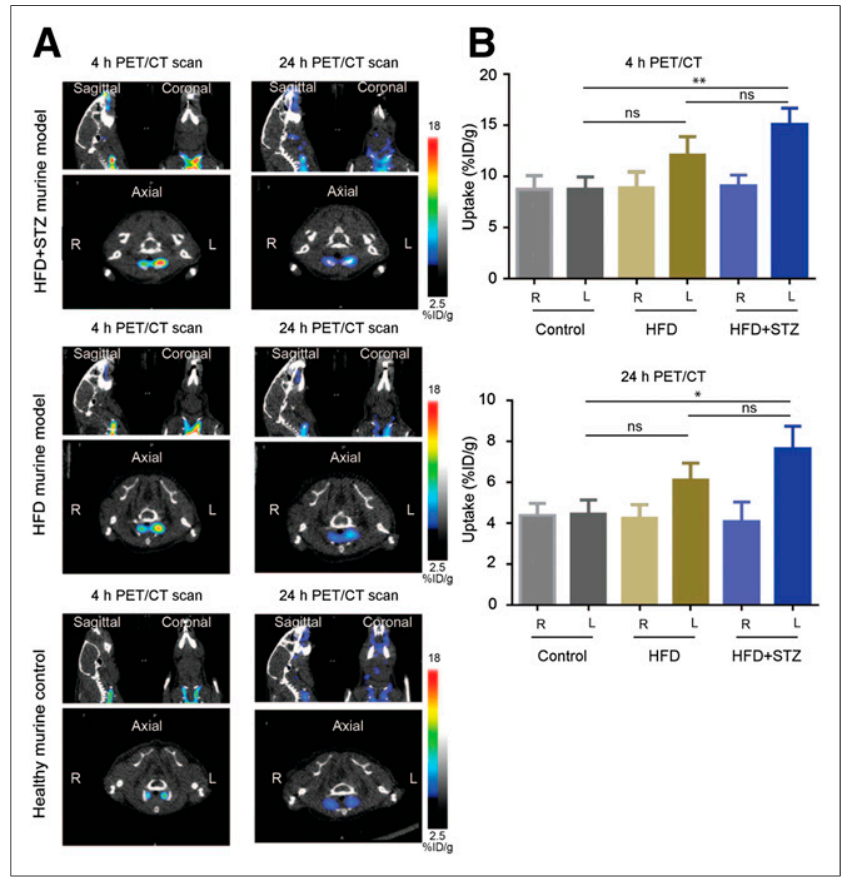

FIGURE 5. Comparison of dual optical/PET imaging probe ${ }^{64} \mathrm{Cu}-\mathrm{BMV} 101$ uptake in HFD + STZ model vs. HFD alone. (A) Noninvasive PET/CT scans of mice with and without ligated carotid arteries. Coronal (top right), sagittal (top left), and axial (bottom, showing left and right). Images are shown for representative HFD + STZ, HFD-alone, and healthy mice imaged at 4 and 24 h. (B) Quantification of 4- and 24-h PET/CT intensity from ligated HFD + STZ, HFD-alone, and nonligated healthy carotid arteries of all mice. Error bars indicate mean + SEM, $n=3,{ }^{\star \star} P<0.005,{ }^{\star} P<0.05$ by $t$ test. 


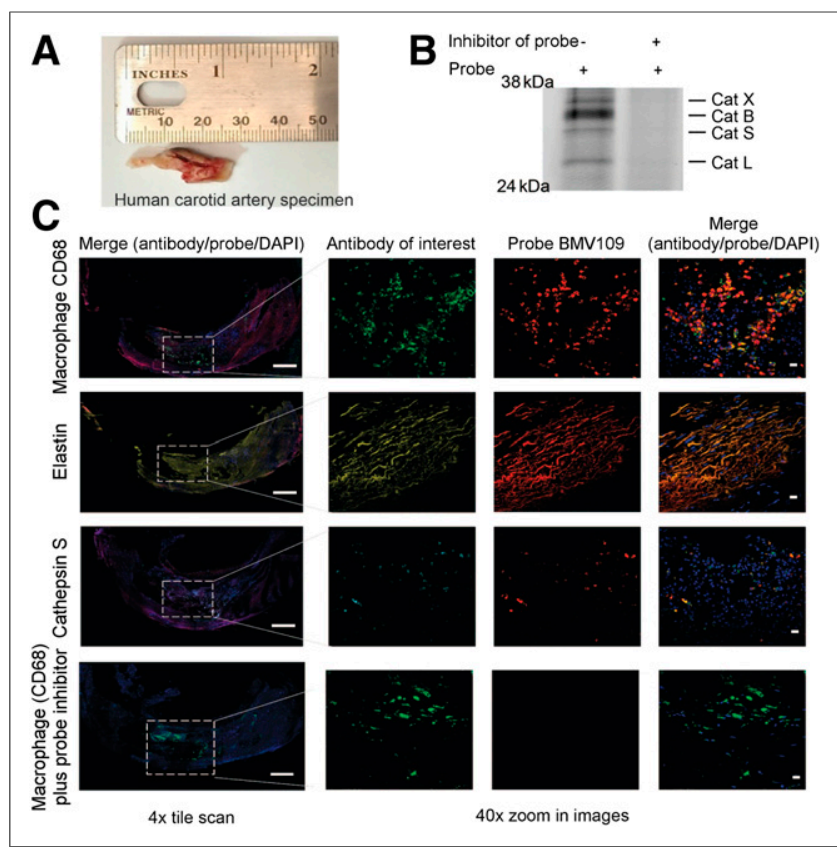

FIGURE 6. Topical application of BMV109 on human carotid endarterectomy sample. (A) Macroscopic specimen. (B) SDS-PAGE analysis followed by flatbed laser scanning to detect probe-labeled cathepsins in carotid artery tissue lysates treated with or without cathepsin inhibitor GB$111 \mathrm{NH}_{2}$. (C) Fresh frozen tissue cross-sections of human carotid artery were labeled with optical probe BMV109 (red) and costained with macrophage activation marker CD68 (green), elastin (yellow), and cathepsin S (cyan). DAPI nuclear stain is shown in blue. Samples were tile-scanned at high resolution to generate full images for which scale bar represents $1 \mathrm{~mm}$. White boxes on full images indicate region that higher magnification images were taken at 40x. Scale bars on zoom images are $10 \mu \mathrm{m}$.

generated are likely to highlight lesions with high levels of inflammatory activity and extracellular matrix remodeling. Increased inflammation and remodeling of the extracellular matrix can predict higher plaque vulnerability; therefore, ABPs targeting protease effector enzymes of these processes may distinguish between stable and vulnerable plaques and should be an area of continued study. Additionally, these ABPs showed efficacy in the murine model of atherosclerosis, in which the animals have comorbid diabetes mellitus. This highlights a significant advantage over the currently used ${ }^{18}$ F-FDG PET/CT imaging agent, which is limited in use because of chronically elevated levels of plasma glucose in diabetic patients. Because diabetes mellitus is a common chronic condition in the population and significantly accelerates the development and severity of cardiovascular disease, it is important that potential noninvasive diagnostic techniques targeting cardiovascular disease have efficacy in diabetic patients.

One limitation of this study is the use of a murine model of cardiovascular inflammation. In these animals, atherosclerotic plaques are rapid in onset and do not fully encompass the complexity and chronic nature of human atherosclerosis. Therefore, to address this shortcoming, we obtained human tissue samples to confirm that our results in the mouse model are consistent with the extent of macrophage involvement observed in human disease. Although more extensive in vivo human studies are needed to further strengthen the validity of our animal model results, our results suggest that the probes perform similarly in both the mouse model and the human tissues. The intensity of the labeling pattern of the arterial wall architecture in fresh frozen tissue samples of both the ligated and the unligated carotid arteries of the diseased hyperlipidemic and diabetic FVB mice, compared with the control animal sections, was somewhat surprising. When these sections were costained with elastin, the signal for the probe and elastin colocalized, consistent with previous reports that cathepsin $\mathrm{S}$ and elastin colocalize, particularly in regions of elastin breaks or remodeling (28). The labeling of these architectural elements in the unligated carotid artery of the diseased animal was particularly interesting, as these sections did not contain plaques. This may reflect the overall proinflammatory state of these animals, which have comorbid diabetes mellitus in addition to the ligated left carotid artery, as well as elevated levels of remodeling along the entire arterial wall.

\section{CONCLUSION}

We demonstrated the use of the optical ABP BMV109 and the dual optical PET/CT ABP BMV101 for noninvasive diagnostic imaging of cardiovascular disease. These probes showed efficacy in a variety of imaging modalities, including FMT and PET/CT, and via topical application of the probe to fresh frozen murine and human tissue sections. Activity-based probes targeting cysteine cathepsins associated with activated monocyte-derived macrophages represent a promising noninvasive technique for the imaging and diagnosis of cardiovascular disease.

\section{DISCLOSURE}

The costs of publication of this article were defrayed in part by the payment of page charges. Therefore, and solely to indicate this fact, this article is hereby marked "advertisement" in accordance with 18 USC section 1734 . This work was funded by U.S. National Institutes of Health grants R01 HL11630702 and R01 EB005011 and received support from a research fellowship from the Manpei Suzuki Diabetes Foundation, a research fellowship from the Fédération Française de Cardiologie, and the Stanford Medical Scientist Training Program. No other potential conflict of interest relevant to this article was reported.

\section{ACKNOWLEDGMENTS}

We thank the Molecular Imaging Program at Stanford and the Stanford Small Animal Imaging Facility for assistance with noninvasive imaging studies. We also thank Pauline Chu from the Department of Pathology at Stanford University for assistance with processing the histology samples and Drs. Ed Harris and Jason T. Lee for assistance with the human arteries.

\section{REFERENCES}

1. Hansson GK. Inflammation, atherosclerosis, and coronary artery disease. $N$ Engl J Med. 2005;352:1685-1695.

2. Libby P, Hansson GK. Inflammation and immunity in diseases of the arterial tree: players and layers. Circ Res. 2015;116:307-311.

3. Davies MJ, Thomas AC. Plaque fissuring: the cause of acute myocardial infarction, sudden ischaemic death, and crescendo angina. Br Heart J. 1985;53: 363-373.

4. Jickling GC, Chaturvedi S. Carotid plaque inflammation in stroke assessed by PET: a burning issue? Neurology. 2014;82:1672-1673.

5. Bentzon JF, Otsuka F, Virmani R, Falk E. Mechanisms of plaque formation and rupture. Circ Res. 2014;114:1852-1866.

6. Usman A, Ribatti D, Sadat U, Gillard JH. From lipid retention to immunemediate inflammation and associated angiogenesis in the pathogenesis of atherosclerosis. J Atheroscler Thromb. 2015;22:739-749. 
7. Chistiakov DA, Bobryshev YV, Nikiforov NG, Elizova NV, Sobenin IA, Orekhov AN. Macrophage phenotypic plasticity in atherosclerosis: the associated features and the peculiarities of the expression of inflammatory genes. Int $J$ Cardiol. 2015;184:436-445.

8. Ley K, Miller YI, Hedrick CC. Monocyte and macrophage dynamics during atherogenesis. Arterioscler Thromb Vasc Biol. 2011;31:1506-1516.

9. Galis ZS, Sukhova GK, Lark MW, Libby P. Increased expression of matrix metalloproteinases and matrix degrading activity in vulnerable regions of human atherosclerotic plaques. J Clin Invest. 1994;94:2493-2503.

10. Sukhova GK, Shi GP, Simon DI, Chapman HA, Libby P. Expression of the elastolytic cathepsins $\mathrm{S}$ and $\mathrm{K}$ in human atheroma and regulation of their production in smooth muscle cells. J Clin Invest. 1998;102:576-583.

11. Vinegoni C, Botnaru I, Aikawa E, et al. Indocyanine green enables near-infrared fluorescence imaging of lipid-rich, inflamed atherosclerotic plaques. Sci Transl Med. 2011;3:84ra45.

12. Garland M, Yim JJ, Bogyo M. A bright future for precision medicine: advances in fluorescent chemical probe design and their clinical application. Cell Chem Biol. 2016;23:122-136.

13. Quillard T, Libby P. Molecular imaging of atherosclerosis for improving diagnostic and therapeutic development. Circ Res. 2012;111:231-244.

14. Rudd JH, Hyafil F, Fayad ZA. Inflammation imaging in atherosclerosis. Arterioscler Thromb Vasc Biol. 2009;29:1009-1016.

15. Alie N, Eldib M, Fayad ZA, Mani V. Inflammation, atherosclerosis, and coronary artery disease: PET/CT for the evaluation of atherosclerosis and inflammation. Clin Med Insights Cardiol. 2015;8:13-21.

16. Dweck MR, Chow MW, Joshi NV, et al. Coronary arterial ${ }^{18} \mathrm{~F}$-sodium fluoride uptake: a novel marker of plaque biology. J Am Coll Cardiol. 2012;59:1539-1548.

17. Menezes LJ, Kayani I, Ben-Haim S, Hutton B, Ell PJ, Groves AM. What is the natural history of ${ }^{18} \mathrm{~F}$-FDG uptake in arterial atheroma on PET/CT? Implications for imaging the vulnerable plaque. Atherosclerosis. 2010;211:136-140.

18. Rudd JH, Myers KS, Bansilal S, et al. ${ }^{18}$ Fluorodeoxyglucose positron emission tomography imaging of atherosclerotic plaque inflammation is highly reproducible: implications for atherosclerosis therapy trials. J Am Coll Cardiol. 2007;50: 892-896.

19. Jezovnik MK, Zidar N, Lezaic L, Gersak B, Poredos P. Identification of inflamed atherosclerotic lesions in vivo using PET-CT. Inflammation. 2014;37:426-434.

20. Rudd JH, Warburton EA, Fryer TD, et al. Imaging atherosclerotic plaque inflammation with $\left[{ }^{18} \mathrm{~F}\right]$-fluorodeoxyglucose positron emission tomography. Circulation. 2002;105:2708-2711.

21. Sadat U, Jaffer FA, van Zandvoort MA, Nicholls SJ, Ribatti D, Gillard JH. Inflammation and neovascularization intertwined in atherosclerosis: imaging of structural and molecular imaging targets. Circulation. 2014;130:786-794.
22. Kitagawa $\mathrm{T}$, Kosuge $\mathrm{H}$, Uchida $\mathrm{M}$, et al. RGD-conjugated human ferritin nanoparticles for imaging vascular inflammation and angiogenesis in experimental carotid and aortic disease. Mol Imaging Biol. 2012;14:315-324.

23. Beer AJ, Pelisek J, Heider P, et al. PET/CT imaging of integrin $\alpha_{\mathrm{V}} \beta_{3}$ expression in human carotid atherosclerosis. JACC Cardiovasc Imaging. 2014;7:178-187.

24. Antonov AS, Kolodgie FD, Munn DH, Gerrity RG. Regulation of macrophage foam cell formation by $\alpha_{\mathrm{V}} \beta_{3}$ integrin: potential role in human atherosclerosis. Am J Pathol. 2004;165:247-258.

25. Hoshiga M, Alpers CE, Smith LL, Giachelli CM, Schwartz SM. $\alpha_{\mathrm{V}} \beta_{3}$ integrin expression in normal and atherosclerotic artery. Circ Res. 1995;77:1129-1135.

26. Lutgens SP, Cleutjens KB, Daemen MJ, Heeneman S. Cathepsin cysteine proteases in cardiovascular disease. FASEB J. 2007;21:3029-3041.

27. Samokhin AO, Lythgo PA, Gauthier JY, Percival MD, Bromme D. Pharmacological inhibition of cathepsin S decreases atherosclerotic lesions in Apoe-/mice. J Cardiovasc Pharmacol. 2010;56:98-105.

28. Figueiredo JL, Aikawa M, Zheng C, et al. Selective cathepsin S inhibition attenuates atherosclerosis in apolipoprotein E-deficient mice with chronic renal disease. Am J Pathol. 2015;185:1156-1166.

29. Blum G, Weimer RM, Edgington LE, Adams W, Bogyo M. Comparative assessment of substrates and activity based probes as tools for non-invasive optical imaging of cysteine protease activity. PLoS One. 2009;4:e6374.

30. Ofori LO, Withana NP, Prestwood TR, et al. Design of protease activated optical contrast agents that exploit a latent lysosomotropic effect for use in fluorescenceguided surgery. ACS Chem Biol. 2015;10:1977-1988.

31. Sanman LE, Bogyo M. Activity-based profiling of proteases. Annu Rev Biochem. 2014;83:249-273.

32. Terashima M, Uchida M, Kosuge $\mathrm{H}$, et al. Human ferritin cages for imaging vascular macrophages. Biomaterials. 2011;32:1430-1437.

33. Blum G, Mullins SR, Keren K, et al. Dynamic imaging of protease activity with fluorescently quenched activity-based probes. Nat Chem Biol. 2005;1:203-209.

34. Withana NP, Garland M, Verdoes M, Ofori LO, Segal E, Bogyo M. Labeling of active proteases in fresh-frozen tissues by topical application of quenched activitybased probes. Nat Protoc. 2016;11:184-191.

35. Withana NP, Ma X, McGuire HM, et al. Non-invasive imaging of idiopathic pulmonary fibrosis using cathepsin protease probes. Sci Rep. 2016;6:19755.

36. Verdoes M, Oresic Bender K, Segal E, et al. Improved quenched fluorescent probe for imaging of cysteine cathepsin activity. J Am Chem Soc. 2013;135:14726-14730.

37. Cutter JL, Cohen NT, Wang J, et al. Topical application of activity-based probes for visualization of brain tumor tissue. PLoS One. 2012;7:e33060.

38. Segal E, Prestwood TR, van der Linden WA, et al. Detection of intestinal cancer by local, topical application of a quenched fluorescence probe for cysteine cathepsins. Chem Biol. 2015;22:148-158. 